\title{
Sepsis kills one million newborns a year: WHO
}

\author{
Cite as: CMAJ 2017 October 10;189:E1272. doi: 10.1503/cmaj.109-5504
}

Posted on cmajnews.com on Sept. 20, 2017.

epsis claims the lives of one million newborns and up to 100000 women during and after pregnancy every year, reported the World Health Organization (WHO). The life-threatening condition arises when the body's response to an infection damages its own tissues and organs. Despite being highly preventable and treatable in early stages, sepsis has been largely overlooked as a major cause of maternal and newborn deaths and complications.

"We have a situation where a lot of attention has gone to other causes of maternal mortality over the years," said Dr. Jeffrey Smith, vice president of technical leadership at Jhpiego, an international women's health nonprofit. In Rwanda and Uganda, "because of their efforts over the years to address causes of mortality such as hemorrhage and preeclampsia, sepsis has become the number one cause of maternal mortality."

Speaking at an international congress on maternal and neonatal sepsis hosted by WHO and the Global Sepsis Alliance on Sept. 12 , Smith noted that global efforts to encourage women to deliver in health facilities may actually be worsening the problem. "Many facilities do not have proper infection prevention protocols; moving into a facility may increase cesarean section rates, which contributes to infection, and the crowding in facilities and lack of staff may contribute" especially if mothers and babies are discharged before sepsis is recognized.

Early recognition and treatment of sepsis is critical to survival, but early signs and symptoms, such as confusion or fever, may not seem serious and are easily missed. Diagnosing the condition in newborns and during pregnancy can be particularly difficult, said Dr. João Paulo Souza of the WHO's Department of Reproductive Health and Research. "When it becomes evident, sepsis may already be severe and advanced with a reduced chance of survival."

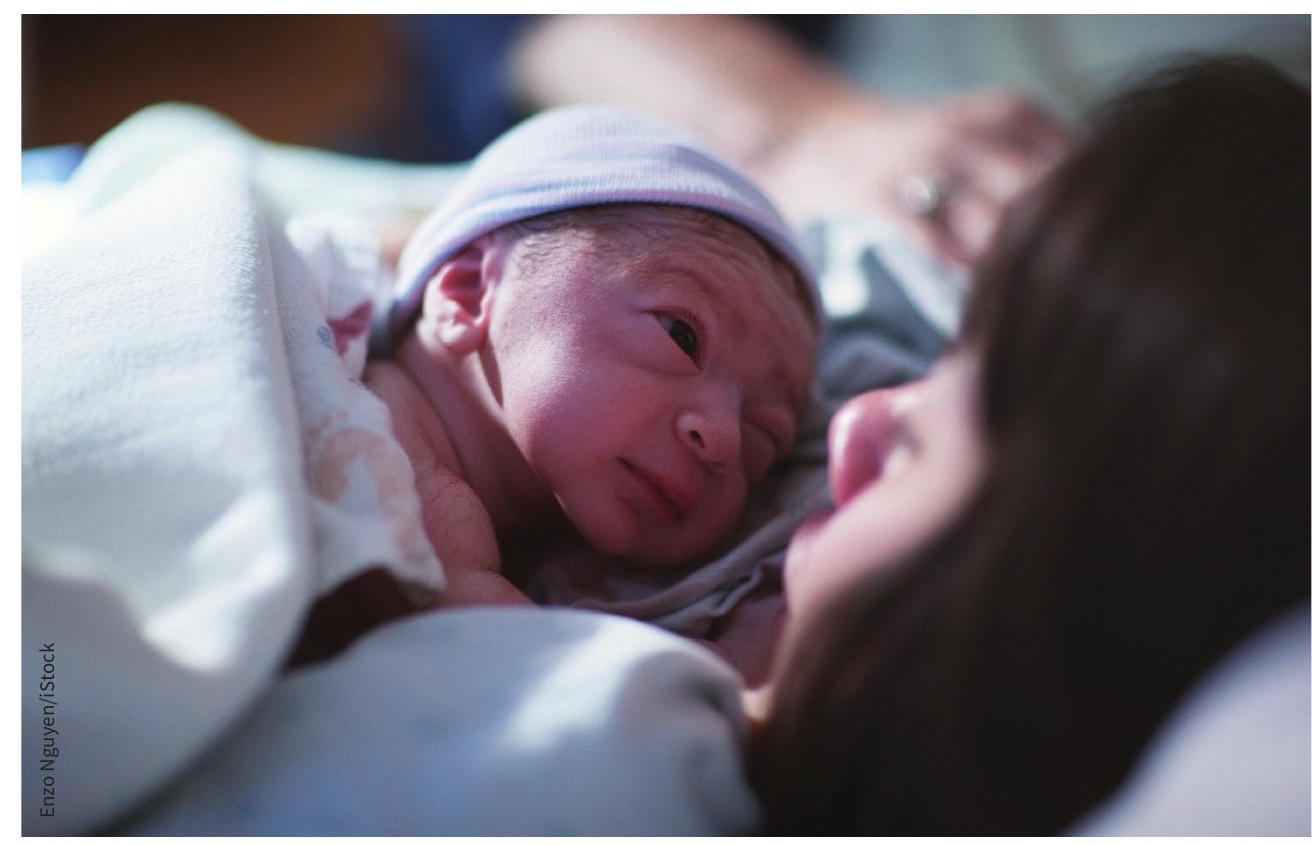

The World Health Organization urges better infection prevention and early diagnosis of sepsis to curb maternal and newborn deaths.

Curbing deaths will require suspecting sepsis much sooner, he said. "Whenever we have a suspected or confirmed infection, we should also look for organ dysfunction, and whenever we have abnormal vital signs or laboratory exams or see a pregnant woman who looks unwell, we should really think sepsis."

Earlier detection and treatment of sepsis in the mother also reduces the risks of transmitting an infection to her baby, said Dr. Zulfiqar Bhutta, codirector of the Centre for Global Child Health at The Hospital for Sick Children in Toronto. Studies also show significant reductions in newborn sepsis deaths associated with the use of clean birth kits, early breastfeeding, skin-to-skin contact ("kangaroo care") and disinfecting the umbilical cord with chlorhexidine, he said. "We have a range of interventions; we have the knowledge."
Whether people are actually using that knowledge is another matter, said Mercedes Bonet, a perinatal health epidemiologist with WHO. "We don't know how maternal sepsis is managed, especially in lower- and middleincome countries," she said. "We don't know if people are implementing protocols proposed for the general adult population or whether these management strategies are effective for maternal sepsis."

Bonet is leading a large study of maternal sepsis in more than 500 health facilities in 54 countries to get a better picture of the prevalence, treatment and prevention of the condition around the world. "The main outcome of the study will be helping people to better identify women at risk of developing sepsis," she said. "We will also have data on antimicrobial resistance that we will use to move forward on this issue."

Lauren Vogel, CMAJ 